\title{
An Integrated Quad-Modality Molecular Imaging System for Small Animals
}

\author{
Yanye Lu ${ }^{1}$, Kun Yang ${ }^{2}$, Kedi Zhou ${ }^{1}$, Bo Pang ${ }^{1}$, Guohe Wang ${ }^{1}$, Yichen Ding ${ }^{1}$, Qiushi Zhang ${ }^{1}$, Hongbin Han ${ }^{1}$, Jiahe Tian ${ }^{3}$, \\ Changhui $\mathrm{Li}^{1}$, and Qiushi Ren ${ }^{1}$ \\ ${ }^{I}$ Department of Biomedical Engineering, College of Engineering, Peking University, Beijing, China; ${ }^{2}$ Department of Control \\ Technology and Instrumentation, College of Quality and Technical Supervision, Hebei University, Baoding, China; and ${ }^{3}$ Department \\ of Nuclear Medicine, The Chinese PLA General Hospital, Beijing, China
}

We developed a novel integrated quad-modality system that included 3 molecular imaging methods (PET, SPECT, and fluorescence molecular imaging [FMI]) and 1 anatomic imaging modality (CT). This system could study various biologic processes in the same animal using multiple molecular tracers. In addition to the technology development, we also discussed the optimization strategy of the imaging protocols. The performance of this system was tested, and the in vivo animal experiment showed its power to trace 3 different molecular probes in living tissues. Our results demonstrated that this system has a great potential for the preclinical study of diseases. Methods: A prototype system integrating PET, SPECT, CT, and a charge-coupled device-based free-space FMI system has been developed. Imaging and fusion capabilities of the system were evaluated by a multimodality phantom. In addition, a mouse disease model with both tumor and inflammation was studied by this system to examine the in vivo performance. The 3 types of molecular probes- ${ }^{18} \mathrm{~F}-\mathrm{FDG}$, [99mTc(HYNIC-3PRGD $)$ (tricine)(TPPTS)]

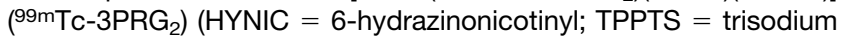
triphenylphosphine-3,3',3"-trisulfonate; $3 \mathrm{PRGD}_{2}=\mathrm{PEG}_{4}-\mathrm{E}\left[\mathrm{PEG}_{4}-\mathrm{C}\right.$ $\left.(\mathrm{RGDfK})]_{2}\right)$, and 3-(triethoxysilyl) propyl-Cy7-entrapped core-crosslinked polymeric micelle (Cy7-entrapped CCPM) nanoparticles-were used to target 3 different biologic processes in the tumor caused by pulmonary adenocarcinoma A549 cells. Moreover, the strategy to optimize multimodal molecular imaging procedure was studied as well, which could significantly reduce the total imaging time. Results: The imaging performance has been validated by both phantom and in vivo animal experiments. With this system and optimized imaging protocol, we successfully differentiated diseases that cannot be distinguished by a single molecular imaging modality. Conclusion: We developed a novel quad-modality molecular imaging system that integrated PET, SPECT, FMl, and CT imaging methods to obtain wholebody multimodality images of small animals. The imaging results demonstrated that this system provides more comprehensive information for preclinical biomedical research. With optimized imaging protocols, as well as novel molecular tracers, this quad-modality system can help in the study of the physiology mechanism at an unprecedented level.

Received Nov. 11, 2013; revision accepted Apr. 29, 2014.

For correspondence or reprints contact either of the following:

Qushi Ren, Department of Biomedical Engineering, College of Engineering,

Peking University, No. 5 Yiheyuan Rd., Beijing, China, 100871.

E-mail: renqsh@coe.pku.edu.cn

Changhui Li, Department of Biomedical Engineering, College of Engineering,

Peking University, No. 5 Yiheyuan Rd., Beijing, China, 100871.

E-mail: chli@pku.edu.cn

Published online Jun. 19, 2014.

COPYRIGHT (c) 2014 by the Society of Nuclear Medicine and Molecular Imaging, Inc.
Key Words: multi-modality imaging; animal imaging; molecular imaging; instrumentation

J Nucl Med 2014; 55:1375-1379

DOI: 10.2967/jnumed.113.134890

$\mathbf{M}$ lecular level in living tissues with high resolution and specificity, sensing diseases at their very early stages. Since it was proposed in the 1990s, molecular imaging has become a key tool in biomedical research and clinical imaging $(1,2)$. Various molecular imaging methods based on different mechanisms, such as radiolabeling and optical labeling, have gained significant progress over the past decades $(3-11)$.

The biologic system involves complex biochemical reactions and the transfer of various cells and molecules. For instance, a breast cancerous lesion could contain several subtype cancer cells, which behave differently in metastasis, metabolism, and angiogenesis. Information on the development and transportation of these cells is essential for accurate diagnosis and effective treatment. In addition, different biologic processes could have serious overlap in molecular reactions. One example is that both cancer and inflammation can cause abnormal metabolic activities, so that tracing glucose metabolism alone is not suitable to separate these 2 distinct diseases. Thus, multiple probes targeting different biomarkers are desired to get important complementary information about these multiple biologic processes. Because different kinds of molecular probes rely on different detecting mechanisms, the integrated multimodal molecular imaging system that can trace multiprobes gains increasing interest and will have great impact for biomedical imaging $(7,12)$.

Up to now, 3 major molecular imaging modalities-PET, SPECT, and fluorescence molecular imaging (FMI) - have been widely used. Relying on radioactive tracers, PET and SPECT have already gained significant clinical applications (13-16). Fluorescence imaging requires optical tracers (e.g., green fluorescence protein) and is favored by researchers because of its versatility, low-cost, and ease of manufacture and use and more importantly because of its unique genetically coding capability. Furthermore, when the image coregistration, safety, reliable handling, and minimization of total imaging time are considered, fluorescence imaging is desired for multimodal molecular imaging in a single system. There are a few existing multimodality animal imaging systems (15) as well as commercial trimodality systems, such as the Inveon (Siemens) (14), FLEX Triumph (Gamma Medica-Ideas), and Albira (Carestream), that have 
provided high system performance with excellent spatial resolution for SPECT and sensitivity for PET. Several articles have discussed the feasibility of combining PET-SPECT and radiology-optical imaging modalities $(7,8,12,17-19)$. To the best of our knowledge, there is no system that has integrated these 3 major molecular imaging modalities.

The development of multimodal molecular imaging technology is not only in hardware development, because different tracers have their own imaging time window and preparation procedures, and signals from various tracers may cause conflicts in detection. Therefore, multimodal molecular imaging also requires a strategy to manage the imaging procedure.

In this article, we presented a novel quad-modality molecular imaging system, which includes PET, SPECT, and FMI and a CT system to provide the anatomic information. The imaging and fusion performance of the system were evaluated by a multimodality phantom. In the animal study, a mouse disease model with both tumor and inflammation was studied in this system. Three types of molecular probes- ${ }^{18} \mathrm{~F}-\mathrm{FDG},\left[{ }^{99 \mathrm{~m}} \mathrm{Tc}\left(\mathrm{HYNIC}-3 \mathrm{PRGD}_{2}\right)(\right.$ tricine)(TPPTS)] $\left({ }^{9} \mathrm{~m}_{\mathrm{Tc}}-3 \mathrm{PRG}_{2}\right)(\mathrm{HYNIC}=6$-hydrazinonicotinyl; TPPTS $=$ trisodium triphenylphosphine-3,3',3"-trisulfonate; $3 \mathrm{PRGD}_{2}=\mathrm{PEG}_{4}-\mathrm{E}\left[\mathrm{PEG}_{4}-\mathrm{c}\right.$ $(\mathrm{RGDfK})]_{2}$ ), and 3-(triethoxysilyl) propyl-Cy7-entrapped core-crosslinked polymeric micelle (Cy7-entrapped CCPM) nanoparticles-were used to target 3 different biologic processes in the animal study. The imaging protocol was carefully optimized to minimize the total imaging time. Our results demonstrated that the quad-modality imaging system, after the optimized procedure, will help researchers to obtain complementary biochemical information for biomedical and preclinical study.

\section{MATERIALS AND METHODS}

\section{Quad-Modality Molecular Imaging System}

The quad-modality imaging system used a modular design — that is, PET, CT, SPECT, and FMI modalities were designed and developed separately (more details are described in the supplemental data). We used a line-type x-ray detector with a helical scan mode in the CT module and a rotary transmission module design. The SPECT system, which has 2 parallel-opposed $\gamma$ cameras to increase the axial field of view that can cover the whole-body area of most mice, was mounted on the same rotating plate with CT (Fig. 1). The $\gamma$ camera had a $22 \times 22$ cerium-doped lutetium-yttrium oxyorthosilicate scintillator array coupled to a Hamamatsu H8500 position-sensitive multianode photomultiplier tube by optical silicone oil. The PET individual module (20) was a fixed ring containing 54 silicon photomultiplier-based detectors. On the end side of the system, a noncontact, full-angle and vertically rotating FMI module was installed. The FMI module can perform not only traditional fluorescence 2-dimensional imaging with both epiillumination and transillumination but also tomographic 3-dimensional imaging. In addition, a transparent animal bed was mounted on a highprecision motor-controlled 1-dimensional translation stage, which carried the animal from one modality to another automatically. The PET, SPECT, and CT modules were set up on one side of the motorcontrolled animal bed, and the FMI module was placed on the other end because that module required strictly light shielding. The entire imaging system can be installed on a $900 \times 1,500 \mathrm{~mm}$ optical table (Fig.1B). There was a custom-designed enclosure (not shown in the figure) that was used to cover the entire system and that also optically shielded the FMI subsystem. In this way, this design not only achieved a compact multimodality system but also avoided signal conflict and optical shielding. Figure 2 presents the overall system design, which consisted of rotary transmission, electronics, power supplies, and several pieces of supporting equipment. The animal bed can be shifted between different modalities, and the multimodality imaging fusion can be done precisely with the coregistered animal position. Geometric

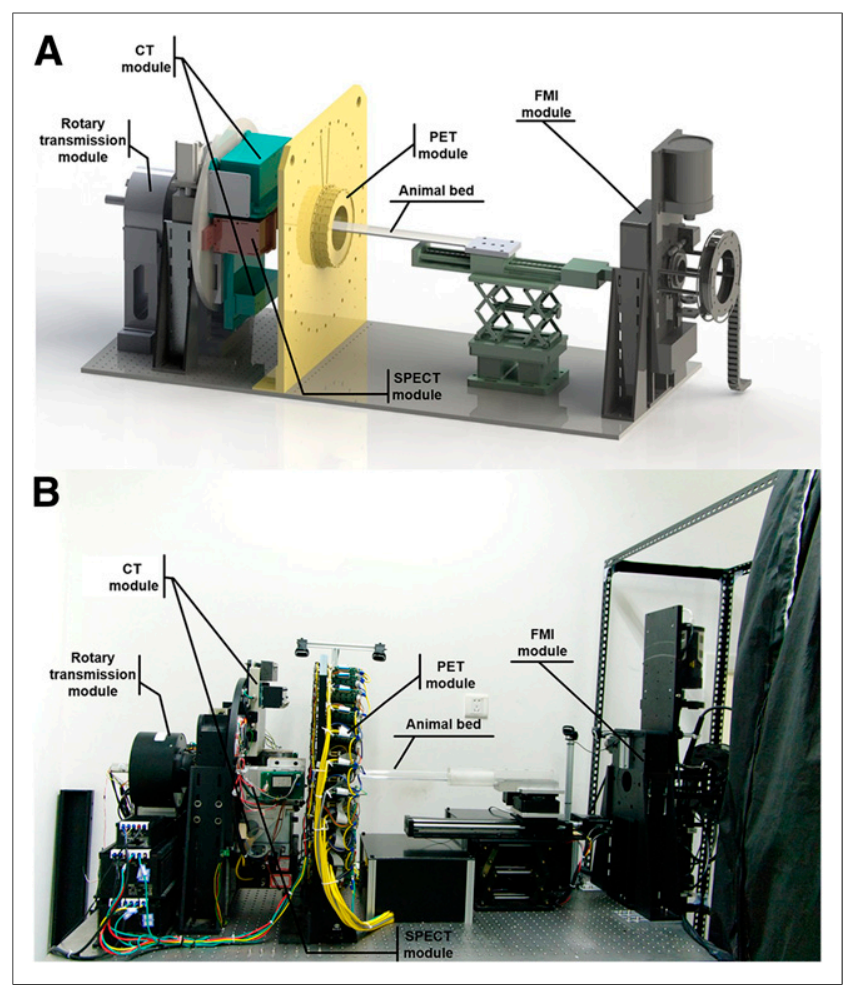

FIGURE 1. (A) Quad-modality imaging system design, which consisted of PET, CT, SPECT, and FMI modules. (B) Photograph of quadmodality imaging prototype system, which was set up on $900 \times 1,500$ $\mathrm{mm}$ optical table.

calibration and image coregistration were processed using fiducial markers on the animal holder.

\section{Optimizing Multimodality Imaging Protocols}

Both phantom and animal experiments were performed to evaluate the imaging capability and fusion performance of the system. Because there are vast differences in the uptake period and imaging technique, each molecular imaging modality requires a specific procedure. Therefore, the overall imaging time can last up to $6 \mathrm{~h}$ in our system without optimization of the imaging protocols. However, long imaging time not only brings difficulties in dynamic study, but also may cause a health hazard for the animals. Thus, optimization of the imaging protocol for multimodal molecular imaging is greatly desired. Given a physiologic or pathologic study case, the following are several primary issues to be considered in assigning the imaging sequence:

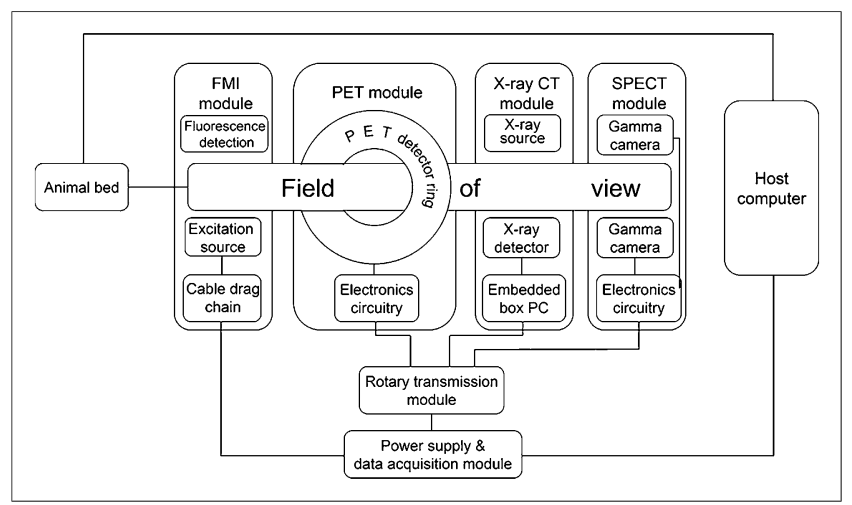

FIGURE 2. Architecture of quad-modality imaging system. $P C=$ personal computer. 
uptake time, preferred imaging period after uptake, imaging operation time, and potential crosstalk with other modalities. In our study, the optimization principle was to perform imaging with short uptake during the long-uptake period of other modalities, as well as separating imaging operations that can cause crosstalk with each other.

\section{Phantom Studies}

The evaluation of imaging and coregistration performance for the prototype system was done by a custom-made multimodality phantom study. The phantom was a solid cylinder (diameter, $30 \mathrm{~mm}$; length, $57 \mathrm{~mm}$ ) having a light scattering property similar to tissue (Fig. 3A). There were 2 holes into which glass tubes (inner diameter, $2.2 \mathrm{~mm}$; length, $50 \mathrm{~mm}$ ) with radiolabeling medicines and fluorescence dyes could be inserted at different insertion depths ( 28 and $45 \mathrm{~mm}$ ) in this cylinder. The bottom zones of the glass tubes were injected with 20 $\mu \mathrm{L}(10 \mu \mathrm{M})$ of indocyanine green as the fluorophore for FMI; the middle section of the 45-mm-insertion-depth glass tube was injected with $7.4 \mathrm{MBq}(0.2 \mathrm{mCi})$ of ${ }^{99 \mathrm{~m}} \mathrm{Tc}-2$-methoxyisobutylisonitrile. The phantom was placed on the animal bed to be first imaged by fluorescence molecular tomography $\left(360^{\circ}\right.$ rotation, scanned for $4 \mathrm{~s} / 10^{\circ}, 36$ angles in total); afterward, the animal bed was shifted to the field of view for the PET/SPECT/CT modules and subjected to SPECT scanning ( $180^{\circ}$ rotation, scanned for $10 \mathrm{~s} / 3^{\circ}, 60$ angles in total). Then, the middle section of the 28 -mm-insertion-depth glass tube was injected with $7.4 \mathrm{MBq}(0.2 \mathrm{mCi})$ of ${ }^{18} \mathrm{~F}-\mathrm{FDG}$ and PET scanning was performed (300- to 650-keV energy window and 12-ns timing window, scanned for $5 \mathrm{~min} /$ per bed position, 3 bed positions). Finally, the phantom was moved to the field of view for the CT scan $(60$ slices, $1.32-\mathrm{mm}$ helical pitch, 360 projection numbers, and 25-ms sampling intervals; $x$-ray tube voltage, $50 \mathrm{keV}$, at $10 \mathrm{~mA}$ ).

FMI data were reconstructed using the algebraic reconstruction technique with a relaxation parameter of 0.1 and 200 iterations. Both SPECT and PET data were reconstructed using the 2-dimensional ordered-subsets expectation maximization algorithms with 10 iterations and 16 subsets; CT data were reconstructed using the filtered backprojection algorithm with a Butterworth filter of order 3 and a cutoff at 0.1 of the Nyquist frequency. All the reconstructed images were coregistered through the geometric calibration to perform image fusion.

\section{In Vivo Animal Studies}

Besides the phantom study, the mouse disease model was studied using this quad-modality imaging system. We induced a xenograft tumor (induced by hypodermic injection of human pulmonary adenocarcinoma A549 cells $\left.\left[2.5 \times 10^{7}, 2.5 \mathrm{~mL}\right]\right)$ and chronic inflammation (induced by hypodermic injection of the proinflammatory substance of Bacillus Calmette-Gu in the right shoulder $[1 \mathrm{mg} / \mathrm{mL}]$ and the right hindlimb $[0.2 \mathrm{~mL}]$ ) of a 16-wk-old, 20-g BALB/C nude mouse. We used ${ }^{18} \mathrm{~F}-\mathrm{FDG}$ for PET imaging, ${ }^{99 \mathrm{~m}} \mathrm{Tc}-3 \mathrm{PRG}_{2}(21)$ for SPECT imaging, and Cy7-entrapped CCPM $(22,23)$ nanoparticles for FMI. ${ }^{18} \mathrm{~F}-\mathrm{FDG}$ traces high-glucose-using cells, ${ }^{99 \mathrm{~m}} \mathrm{Tc}-3 \mathrm{PRGD}_{2}$ is able to target integrin $\alpha_{\mathrm{v}} \beta_{3}$-positive tumors, and Cy7-entrapped CCPM nanoparticles can preferentially accumulate in the tumor site because of the enhanced permeability and retention effect. The mouse was intravenously injected with $0.2 \mathrm{~mL}\left(1.5 \times 10^{15}\right.$ particles/mouse $)$ of Cy7entrapped CCPM nanoparticles and $37.0 \mathrm{MBq}(1.0 \mathrm{mCi})$ of ${ }^{99 \mathrm{~m} T \mathrm{c}-}$ $3 \mathrm{PRG}_{2}$ via the tail vein. After a 50-min uptake period, the mouse underwent anesthesia (2.50\% isoflurane in oxygen [1.5 1/min] for inducing anesthesia and then down to $1.00 \%$ isoflurane in oxygen [0.8 1/ $\mathrm{mL}$ ] for maintenance). The mouse was scanned with SPECT $\left(180^{\circ}\right.$ of rotation, $20 \mathrm{~s} / 3^{\circ}, 60$ angles; duration, $20 \mathrm{~min}$ ) after the 60 -min uptake period of ${ }^{99} \mathrm{~m} \mathrm{Tc}-3 \mathrm{PRG}_{2}$. Then the mouse was intravenously injected with $37.0 \mathrm{MBq}(1.0 \mathrm{mCi})$ of ${ }^{18} \mathrm{~F}-\mathrm{FDG}$ via the tail vein, and the anesthesia gas was increased to $2.5 \%$ isoflurane in oxygen during the injection and down to $1.00 \%$ after the injection. At the end of the 120-min uptake period of Cy7-CCPM nanoparticles, a helical CT scan (60 slices; $1.5-\mathrm{m}$ helical pitch; 360 projection numbers and $25-\mathrm{ms}$ sampling intervals; $\mathrm{x}$-ray tube voltage, $50 \mathrm{keV}$; tube current, $10 \mathrm{~mA}$ ) was obtained. Then, the mouse underwent FMI (epiillumination mode; integration times, $5 \mathrm{~s}$ ). Both CT imaging and FMI were performed during the 60 -min ${ }^{18} \mathrm{~F}$-FDG uptake period. After the period, the mouse was PETscanned (300- to 650-keV energy window, 12-ns timing window, $10 \mathrm{~min} /$ bed position) for $40 \mathrm{~min}$ of 4 bed positions. The whole animal experimental procedure is illustrated in Figure 4B. After the examination, the mouse was sacrificed, and the tumor and inflammation tissues were excised, fixed in $4 \%$ paraformaldehyde, and embedded in paraffin. Hematoxylin and eosin staining was performed on 5 - $\mu \mathrm{m}$-thick tissue sections.

The PET/SPECT/CT images were reconstructed as aforementioned. Because the subcutaneous tumor was located in a shallow region of the body, only the 2dimensional fluorescence imaging was given by using the epiillumination FMI. All the reconstructed images were coregistered automatically to perform image fusion. Animal studies were performed in accordance with the guidelines from the Peking University Laboratory Animal Centre and protocols approved by this institution.

\section{RESULTS}

\section{Performance Evaluation by Phantom Study}

Figure 3 shows the imaging results, including images by individual modalities and coregistered images. Both PET and SPECT provided-high quality images without observable crosstalk artifacts. In order to demonstrate the spatial resolution, a length of bubble $(3 \mathrm{~mm})$ separating 2 PET medicine drops was made, and this small gap was clearly distinguished in PET images. In ad-

dition, FMI also successfully reconstructed
FIGURE 3. Imaging results of multimodality phantom. CT, PET, SPECT, fluorescence molecular, and quad-modality fusion images are presented. In results, quality of SPECT images has not been affected by crosstalk from positron-emitting radionuclides. Maximum-intensity-projection rendering of fusion images $(C)$ demonstrated coregistration performance. Right, middle, and left colorbar correspond to PET, SPECT, and fluorescence molecular images, respectively. max = maximum. 


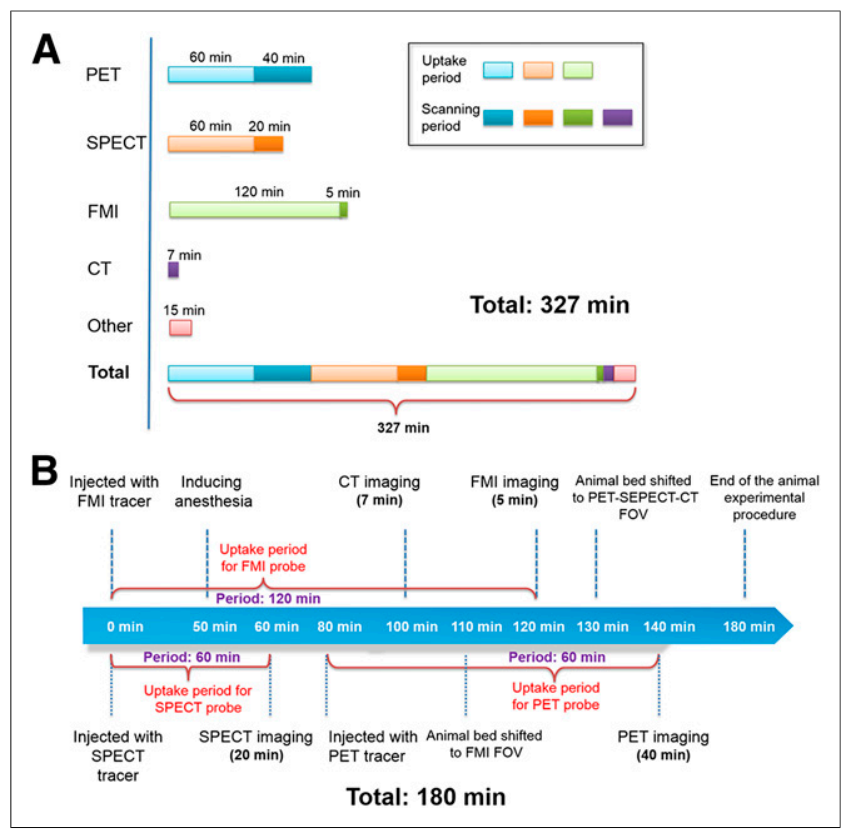

FIGURE 4. Timeline schematic of whole animal experimental procedure. (A) Typical quad-modality imaging procedure in this animal study takes more than $320 \mathrm{~min}$, considering time needed for drug metabolism. (B) After optimization of imaging protocol, total time was reduced to $180 \mathrm{~min}$, as well as avoiding crosstalk issue.

the fluorophores deep in the phantom. In Figure 3C, the maximumintensity-projection rendering of the fused images demonstrated the accurate coregistration. The imaging results indicated that our hybrid multimodality imaging system could provide appropriate imaging and accurate image fusing.

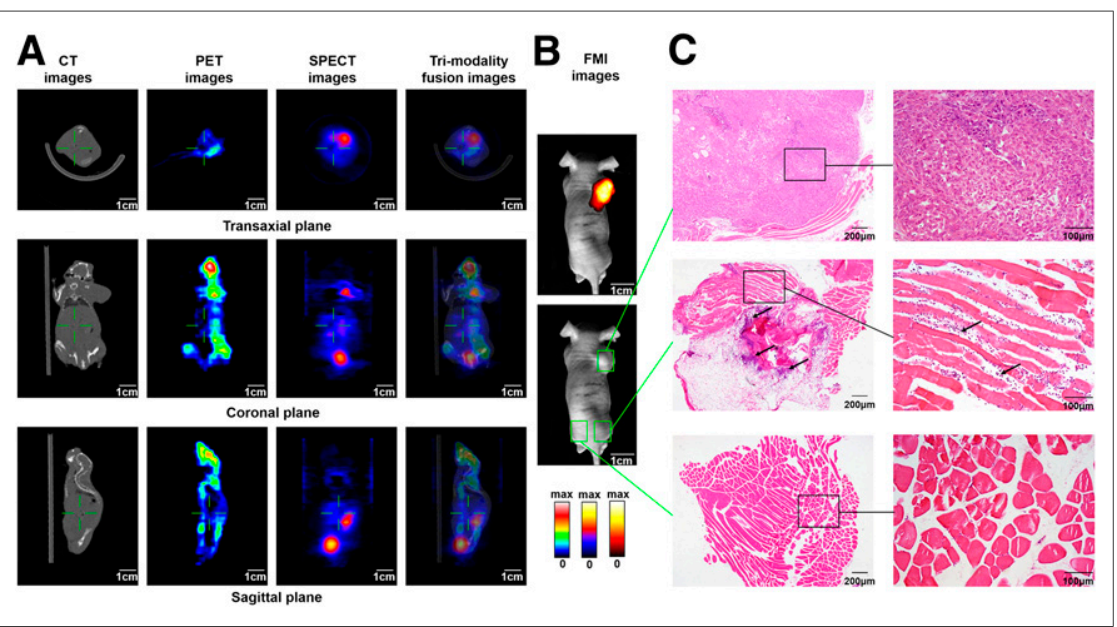

FIGURE 5. Quad-modality in vivo animal study. Complementing CT scanning results, PET images showed high-radioactivity accumulation in brain, right shoulder, and both legs. In SPECT images, radioactivity accumulation of ${ }^{99 \mathrm{~m} T c-3 P R G D}$ in right shoulder, abdomen, and area of bladder was evident. There was only area of right shoulder that had great accumulation of Cy7entrapped CCPM nanoparticles. We were able to identify tumor location by complementary information obtained from PET-SPECT-CT fusion images, and in addition, FMI results also confirmed tumor location. (C) Histology (upper) confirmed tumor area of right shoulder, with high cell proliferation observed with high tracer uptake in PET, SPECT, and fluorescence molecular images. Histology (middle) confirmed inflammation area of right leg, with large number of inflammatory cells such as neutrophils (arrow) showing increased ${ }^{18} \mathrm{~F}-\mathrm{FDG}$ accumulation in PET images. Right, middle, and left colorbar correspond to PET, SPECT, and fluorescence molecular images, respectively. $\max =$ maximum.

\section{Imaging Procedure for In Vivo Study}

The imaging procedure consisted of tracer injections, periods of mized the imaging protocol to minimize the total imaging time. In this work, we focused on the multimodal molecular imaging nuclides used in PET imaging create a serious crosstalk problem affecting the quality of the SPECT imaging, and the imaging time will be much longer if the experiments start with PET before SPECT due to the half-life period of PET radionuclides. In addition, because of the relatively long uptake period for fluorescence tracers, other modalities can be performed earlier than FMI. On the basis of these considerations, Figure 4B presented our optimized imaging protocol.

\section{In Vivo Animal Studies}

Figure 5 shows the multimodality and multiprobe imaging results. Complementing the CT results, the PET images showed high radioactivity accumulation in the brain, right shoulder, and right legs. In SPECT images, the radioactivity accumulation of ${ }^{99 \mathrm{~m} T c-}$ $\mathrm{RGD}_{2}$ primarily occurred in the right shoulder, abdomen, and $4 \mathrm{~B}$, only the area of the right shoulder had a significant accumulaon of Cy-entrapped CCPM nanoparticles. SPECT-CT fusion images. On the other hand, fluorescence molecular images showed the correct area that was suggestive of tumor, but the imaging resolution was low. Complementing FMI with radioactive molecular imaging significantly improved both specificity and resolution. This result successfully demonstrated the superior power of multimodal molecular imaging.

Moreover, by following the optimized imaging procedure, the entire imaging time for this in vivo study, including preparation and imaging, took only $3 \mathrm{~h}$. However, the procedure can last up to $6 \mathrm{~h}$ just to sum all imaging times from each individual modality. The significant reduction in imaging time is essential to study dynamic physiologies. The imaging time can be reduced even further after system improvement.

\section{DISCUSSION}

Successful multimodal molecular imaging requires innovations in both technology development and imaging protocols. In this study, we not only developed the first quadmodality imaging system for preclinical in vivo molecular imaging studies, but also provided a strategy to optimize the imaging protocol. The performance of the system and image coregistration have been validated by both phantom and in vivo animal experiments.

In the animal disease model study, the quad-modality imaging system successfully found tumors and acquired images non- 
invasively and in vivo. The 4 modalities of PET, SPECT, FMI, and CT can provide more comprehensive information for molecular imaging studies, improving the overall specificity. In our study, the image fusion of a tumor xenograft mouse (Fig. 5) showed complementary information that helped to differentiate between the tumor location and the inflammation location. These findings were confirmed by ex vivo histology.

This quad-modality system also has a great potential for cancer subtype diagnosis. Metabolism, metastasis, and neovascularization are the 3 most important characteristics of the pathologic behavior of cancer cells. According to recent studies, many cancers have various subtypes, and not all of them are aggressive. Differentiation of the subtypes of cancer cells has an important role in the study of the pathology of cancer as well as its treatment (24-26). Traditionally, molecular biology approaches such as immunohistochemistry are used for revealing the biologic behavior of cancer cells; however, recent molecular imaging approaches-which allow for noninvasive in vivo imaging and quantification of biologic processes such as metabolism, metastasis, and neovascularization-are more and more widely used in these studies. With the quad-modality system, 3 different tracers for PET, SPECT, and FMI can trace these 3 types of cells with high specificity and accurate image fusion. However, this cannot be done with any current single system $(15,18)$.

In addition, this quad-modality imaging system was modulardesigned. All 4 modalities were coaxial-aligned, and a transparent animal holder controlled by a high-precision translational and rotational stage can deliver the animal from one modality to another. Our system can be used as single, dual-, tri-, or quad-modalities such as CT, PET/CT, SPECT/CT, FMI-CT, PET-SPECT-CT, or PETSPECT-FMI-CT, based on different research requirements.

Multimodal molecular imaging generally required an extended time. For instance, in our system, SPECT typically needs to wait for $60 \mathrm{~min}$ after the injection of medicine, and it took another 2030 min to finish the scanning. In addition, different imaging modalities might have conflicts, such as the potential risk of crosstalk between PET and SPECT. However, summation of operation time by all 3 molecular imaging modalities, as well as the uptake time of radioactive medicine, will be about $6 \mathrm{~h}$. Thus, the procedure of multimodal molecular imaging must be carefully optimized to minimize the total scanning time. To avoid crosstalk, the PET tracer was injected after the SPECT scan in our system. In addition, long-lasting fluorescence tracers could be injected the day before the experiment. Finally, we achieved a 3-h imaging time. The total length of the scanning time can be even shortened by performance-improvement of the imaging system. For example, we can inject both radiolabeling tracers simultaneously using a better SPECT detector that can differentiate signals from PET tracer (16). In addition to the technical development, the in vivo animal study is our next primary research work.

\section{CONCLUSION}

We developed a novel quad-modality molecular imaging system that integrated PET, SPECT, FMI, and CT imaging modalities to obtain whole-body multimodality molecular images of small animals. The optimization in both system setup and imaging procedure are all carefully considered. We demonstrated that this system provides more comprehensive information for preclinical biomedical research. Using optimized imaging protocols and novel molecular tracers, this quad-modality system can help people understand the physiology mechanism on an unprecedented level.

\section{DISCLOSURE}

The costs of publication of this article were defrayed in part by the payment of page charges. Therefore, and solely to indicate this fact, this article is hereby marked "advertisement" in accordance with 18 USC section 1734. This work is supported by The National Key Instrumentation Development Project (2011YQ030114), The National Basic Research Program of China (973 Program, 2011CB707500), the National Natural Science Foundation of China (11104058), and the Natural Science Foundation of Hebei Province (A2011201155). No other potential conflict of interest relevant to this article was reported.

\section{REFERENCES}

1. Bushberg JT, Seibert JA, Leidholdt EM, Boone JM. The Essential Physics of Medical Imaging. Philadelphia, PA: Wolters Kluwer Health; 2011.

2. de Kemp RA, Epstein FH, Catana C, Tsui BMW, Ritman EL. Small-animal molecular imaging methods. J Nucl Med. 2010;51(suppl 1):18S-32S.

3. Beyer T, Townsend DW, Brun T, et al. A combined PET/CT scanner for clinical oncology. J Nucl Med. 2000;41:1369-1379.

4. Townsend DW. Multimodality imaging of structure and function. Phys Med Biol. 2008;53:R1-R39.

5. Judenhofer MS, Wehrl HF, Newport DF, et al. Simultaneous PET-MRI: a new approach for functional and morphological imaging. Nat Med. 2008;14:459-465.

6. Zaidi H, Prasad R. Advances in multimodality molecular imaging. J Med Phys. 2009;34:122-128.

7. Cherry SR. Multimodality imaging: beyond PET/CT and SPECT/CT. Semin Nucl Med. 2009;39:348-353.

8. Ale A, Ermolayev V, Herzog E, Cohrs C, de Angelis MH, Ntziachristos V. FMTXCT: in vivo animal studies with hybrid fluorescence molecular tomographyX-ray computed tomography. Nat Methods. 2012;9:615-620.

9. Zhang Q, Brukilacchio TJ, Li A, et al. Coregistered tomographic x-ray and optical breast imaging: initial results. J Biomed Opt. 2005;10:024033.

10. Hasegawa BH, Stebler B, Rutt BK, et al. A prototype high-purity germanium detector system with fast photon-counting circuitry for medical imaging. Med Phys. 1991;18:900-909.

11. Lang TF, Hasegawa BH, Liew SC, et al. Description of a prototype emission transmission computed tomography imaging system. J Nucl Med. 1992;33:1881-1887.

12. Stout DB, Zaidi H. Preclinical multimodality imaging in vivo. PET Clin. 2008;3:251-273.

13. Davis SC, Pogue BW, Springett R, et al. Magnetic resonance-coupled fluorescence tomography scanner for molecular imaging of tissue. Rev Sci Instrum. 2008;79:064302.

14. Magota K, Kubo N, Kuge Y, Nishijima K-i, Zhao S, Tamaki N. Performance characterization of the Inveon preclinical small-animal PET/SPECT/CT system for multimodality imaging. Eur J Nucl Med Mol Imaging. 2011;38:742-752.

15. Wang G, Zhang J, Gao H, et al. Towards omni-tomography-grand fusion of multiple modalities for simultaneous interior tomography. PLOS ONE. 2012;7:e39700.

16. Goorden MC, van der Have F, Kreuger R, et al. VECTor: a preclinical imaging system for simultaneous submillimeter SPECT and PET. J Nucl Med. 2013;54:306-312.

17. Cherry SR. Multimodality in vivo imaging system: twice the power or double the trouble? Annu Rev Biomed Eng. 2006;8:35-62.

18. Culver J, Akers W, Achilefu S. Multimodality molecular imaging with combined optical and SPECT/PET modalities. J Nucl Med. 2008;49:169-172.

19. Liu H, Ren G, Miao Z, et al. Molecular optical imaging with radioactive probes. PLOS ONE. 2010;5:e9470.

20. Lu Y, Yang K, Zhou K, Zhang Q, Pang B, Ren Q. Development of a SiPM-based PET imaging system for small animals. Nucl Instrum Meth A. 2014;743:30-38.

21. Jia B, Liu Z, Zhu Z, et al. Blood clearance kinetics, biodistribution, and radiation dosimetry of a kit-formulated integrin $\alpha \mathrm{v} \beta 3$-selective radiotracer ${ }^{99 \mathrm{~m}} \mathrm{Tc}-3 \mathrm{PRGD}$ 2 in non-human primates. Mol Imaging Biol. 2011;13:730-736.

22. Yang Z, Zheng S, Harrison WJ, et al. Long-circulating near-infrared fluorescence core-cross-linked polymeric micelles: synthesis, characterization, and dual nuclear/optical imaging. Biomacromolecules. 2007;8:3422-3428.

23. Yang Z, Leon J, Martin M, et al. Pharmacokinetics and biodistribution of nearinfrared fluorescence polymeric nanoparticles. Nanotechnology. 2009;20:165101.

24. Wang H, Zhang J, Tian J, et al. Using dual-tracer PET to predict the biologic behavior of human colorectal cancer. J Nucl Med. 2009;50:1857-1864.

25. Pantel K, Brakenhoff RH, Brandt B. Detection, clinical relevance and specific biological properties of disseminating tumour cells. Nat Rev Cancer. 2008;8:329-340.

26. Powell AA, Talasaz AH, Zhang H, et al. Single cell profiling of circulating tumor cells: transcriptional heterogeneity and diversity from breast cancer cell lines. PLoS ONE. 2012;7:e33788. 